\title{
Comparing bone mineral density in postmenopausal women with and without vertebral fracture and its value in recognizing high-risk individuals
}

B. Heidari, ${ }^{1}$ S. Hoshmand, ${ }^{1}$ K. Hajian ${ }^{2}$ and P. Heidari ${ }^{3}$

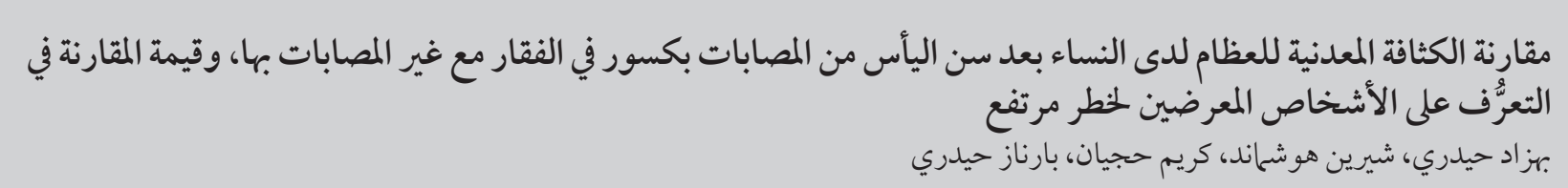

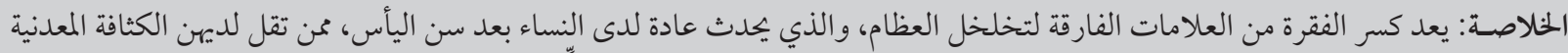

ABSTRACT Vertebral fracture, the hallmark of osteoporosis, usually occurs in postmenopausal women with low bone mineral density (BMD). The aim this study was to determine which BMD parameter can best predict women at high risk of fracture. BMD values at the spine and femoral neck were compared in 34 women with vertebral fracture and 34 controls. Mean BMD $\left(\mathrm{g} / \mathrm{cm}^{2}\right), T$-score, and Z-score values at both femoral neck and spine were significantly lower in the fracture than the nonfracture group. Z-score was more sensitive than BMD T-score for detecting low bone mass. T-score was not sensitive enough to identify low BMD in the spine, whereas the femoral neck T-score could recognize women at high risk of fracture with higher accuracy.

Comparaison de la densité minérale osseuse chez des femmes ménopausées présentant ou non une fracture vertébrale et sa valeur dans l'identification d'individus à haut risque

RÉSUMÉ La fracture vertébrale, caractéristique de l'ostéoporose, survient généralement chez la femme ménopausée ayant une faible densité minérale osseuse (DMO). Cette étude visait à déterminer quel paramètre de la DMO était susceptible d'identifier au mieux les femmes qui pourraient être à haut risque de fracture. Des mesures dela DMO réalisées au niveau de la colonne vertébrale et du col du fémur chez 34 femmes présentant une fracture vertébrale et 34 témoins ont été comparées. Par rapport au groupe témoin, les valeurs moyennes de la DMO $\left(\mathrm{g} / \mathrm{cm}^{2}\right)$, du T-score et du Z-score relevées sur le col du fémur et la colonne vertébrale étaient considérablement inférieures chez les femmes souffrant de fractures. Le Z-score s'est avéré plus sensible que le T-score de la DMO pour détecter une faible densité osseuse. II est apparu que le T-score n'était pas assez sensible pour identifier une faible DMO de la colonne vertébrale, alors qu'il permettait d'identifier avec une meilleure précision les femmes présentant un risque élevé de fracture du col du fémur.

${ }^{7}$ Department of Internal Medicine; ${ }^{2}$ Department of Social Medicine, Shaheed Beheshti Hospital, Babol University of Medical Sciences, Babol, Islamic Republic of Iran (Correspondence to B. Heidari: heidaribeh@yahoo.com).

${ }^{3}$ Faculty of Medicine, Islamic Azad University, Tehran, Islamic Republic of Iran.

Received: 10/01/09; accepted: 26/02/09 


\section{Introduction}

Vertebral fracture is the most common osteoporotic fracture and also a hallmark of postmenopausal osteoporosis. It is associated with back pain, disability, functional limitation and decreased quality of life [1-6]. Bone mineral density (BMD) is an important predictor of future fractures. Low BMD at the spine or femoral neck in postmenopausal women has been shown in several prospective studies to be associated with increased risk of vertebral fracture [7-10]. In the elderly population, however, factors other than BMD, such as age, might play a role in the development of fracture, because older patients are much more susceptible to fractures at any given BMD than are younger patients, perhaps due to the quality of ageing bone [4,5,11-13].

Several studies havefound a relationship between increasing risk of vertebral fracture and decreasing BMD values in women $[14,15]$. There is also a strong association between age and femoral neck BMD with vertebral fracture [16]. Nevertheless, many postmenopausal women with fractured vertebra are not osteoporotic, so BMD measurements cannot accurately predict individuals who will develop fractures. This issue has been attributed to discordance between the site of BMD measurement and the site of fractures; low sensitivity of the BMD T-score criterion; inconsistency between BMD measurement and the time of the fracture; and false elevation of BMD values due to the presence of degenerative changes at the site of $\mathrm{BMD}$ measurement $[17,18]$. Given the burden of vertebral fractures in the elderly, detection of low bone mass for prevention offractures is important. At present, the BMD T-score (which measures how much bone mass deviates from the average bone mass of a healthy adult) is used as a tool for evaluation of bone mass as well as for fracture assessment, as recommended by the World Health Organization (WHO) [19].
We hypothesized that the magnitude of differences in the BMD of spine or femoral neck would be comparable in women with and without fractures irrespective of the BMD parameter used. The aim of this study was to determine which parameter of BMD is more sensitive in diagnosis of low BMD and can more accurately identify women susceptible to vertebral fractures.

\section{Methods}

The study compared postmenopausal women with and without vertebral fractures with respect to bone mass at the spine and femoral neck.

\section{Sample}

A sample of 34 postmenopausal women with back pain and vertebral fracture and 34 aged-matched postmenopausal women with back pain but without vertebral fracture entered the study. The study patients attended or were referred to an outpatient medical clinic from the general population of Babol, Islamic Republic of Iran. The women who met the selection criteria were selected sequentially over a 6-month period from June to December 2007.

Only patients with recent back pain who had pain and tenderness at the lumbar spine with symptom duration of less than 6 weeks were included. Those with previous spinal surgery, malignan$\mathrm{cy}$, traumatic fracture or inflammatory skeletal disease and patients on steroids or anti-osteoporosis treatment were excluded. Patients with fractures at the site of BMD assessment in the lumbar spine were also excluded from the study.

This study was approved by the ethics committee of Babol University of Medical Sciences.

\section{Data collection}

Data regarding demographic and clinical characteristics were collected by interview and questionnaire. Clinical, radiographic and bone densitometry data were provided by clinical examination, X-ray examination and BMD measurements.

At the first visit all patients underwent a complete clinical examination, spinal X-ray, and BMD measurements at the lumbar spine (L2-L4) and femoral neck by dual X-ray absorptiometry (DXA) with a single Dorland densitometer. BMD was evaluated based on a reference database provided by the manufacturer.

The mean duration of back pain prior to spinal radiography and themean duration between diagnosis of vertebral fracture and BMD measurement was $<2$ weeks in about $80 \%$ of patients and $<6$ weeks in the remainder.

Clinical vertebral fracture was diagnosed by history, clinical examination and spinal X-ray. Vertebral fracture was assessed by the Genant semiquantitative method in a lateral-view spinal Xray by an experienced physician [20]. In this method, the extent of the vertebral height reduction and morphological changes are assessed visually and scored using a grading scale to differentiate fracture from other nonfracture deformities. Thoracic and lumbar vertebra from T4-L4 were graded based on the approximate degree of height reduction. Vertebrae with a 20\%-25\% or greater reduction in anterior, middle and/or posterior height relative to adjacent vertebrae of normal appearance were considered as fractured vertebrae. Women with nonosteoporotic vertebral deformity and traumatic fractures were excluded from the study.

Osteoporosis was confirmed according to WHO criteria [19], defined as BMD value $>2.5$ standard deviation (SD) below the young normal mean BMD (T-score <-2.5).

\section{Analysis}

For the statistical analysis patients with vertebral fractures (at least 1 fractured vertebra) were compared with those without fractures in terms of bone 
density values (BMD, T-score and Zscore (which measures how much bone mass deviates from the average bone mass of people the same age and sex), frequency of osteoporosis at the spine or femoral neck regions and duration of menopause. The differences were expressed as mean percentage difference of the fracture group from the nonfracture group.

The association between osteoporosis and vertebral fracture was determined by calculation of odds ratio (OR) and its 95\% confidence interval (CI) using the chi-squared test. Student $t$-test was used for comparison of BMD values.

Receiver operating characteristics (ROC) curve was applied to evaluate the trade-off between sensitivity and 1-specificity for various cut-off points of BMD values to assess the diagnostic accuracy of BMD parameters and age in discriminating between the fracture and the nonfracture group. Diagnostic accuracy of BMD parameters were compared with respect to values of the area under the curve (AUC) and its 95\% CI in comparison with the chance level (AUC $=50 \%)$. The impact of age on diagnostic accuracy was assessed by comparison of AUC values between 2 age groups ( $<65$ and $\geq 65$ years).

Statistical analysis was performed using SPSS software, version 10.

\section{Results}

The mean age of the study patients was 66.6 (SD 8.4) years and the mean duration of menopause was 17.9 (SD 9.2) years. Two-thirds of women (64.7\%) were aged over 65 years. Vertebral fracture was associated with acute back pain in $84.6 \%$ of cases versus $23.5 \%$ of those without fractures.

As shown in Table 1 the mean age of patients with vertebral fractures was 67.5 (SD 9.7) years and those without fractures was 65.6 (SD 7.0) years (not significant). The mean duration of menopause in patients with and without fractures was 19.6 (SD 10.4) years and 16.2 (SD 7.7) years respectively (not significant). The mean BMD (g/ $\left.\mathrm{cm}^{2}\right)$, T-score and Z-score values at both the femoral neck and spine regions in the fracture group were significantly lower than in the nonfracture group (Table 1).

In the fracture group the mean spine BMD T-score was 35\% lower whereas the spine Z-score was 58\% lower than in the nonfracture group. The corresponding values for femoral neck $\mathrm{T}$ score and Z-score were 24\% and 100\% respectively. The size of the difference between the 2 groups varied with age and site of BMD measurements. In the fracture group aged $<65$ years the corresponding differences between spine
T-score and Z-score were 59\% and 92\% respectively and in patients $\geq 65$ years were $20 \%$ and $51 \%$. In the femoral neck the corresponding values for femoral neck $\mathrm{T}$-score and Z-score in women aged $<65$ years were $30 \%$ and $160 \%$ and in women aged $\geq 65$ years were $19 \%$ and $71 \%$ respectively.

Vertebral fracture developed in $61.7 \%$ of patients with spinal osteoporosis versus $37.1 \%$ patients without spinal osteoporosis $(\mathrm{OR}=2.7,95 \% \mathrm{CI}$ : 1.3-7.2, $P<0.05)$. The corresponding values for patients with and without femoral neck osteoporosis were $60.0 \%$ and $25.0 \%$ respectively $(\mathrm{OR}=4.6,95 \%$ CI: $1.4-14.6, P=0.015)$.

Femoral neck osteoporosis was more strongly associated with vertebral fracture in women aged $<65$ years versus those aged $\geq 65$ years $(\mathrm{OR}=10.0$, 95\% CI: 1.4-69.2, $P=0.036)$ whereas the association between spinal osteoporosis and vertebral fracture was not different comparing patients aged $<65$ years with older patients. The presence of osteoporosis at both sites, femoral neck and spine, increased the risk of vertebral fracture by almost 6-fold (OR $=5.8,95 \%$ CI: $1.5-21.9, P<0.011)$.

Comparison of bone mass at fracture and nonfracture sites with BMD $\mathrm{T}$-score and Z-score gave different results with respect to BMD parameters. Based on BMD T-scores, low bone

\begin{tabular}{|c|c|c|c|c|c|}
\hline \multirow[t]{2}{*}{ Variable } & \multicolumn{2}{|c|}{$\begin{array}{l}\text { Vertebral fracture present } \\
\qquad(n=34)\end{array}$} & \multicolumn{2}{|c|}{$\begin{array}{l}\text { Vertebral fracture absent } \\
\qquad(n=34)\end{array}$} & \multirow[t]{2}{*}{$P$-value ${ }^{a}$} \\
\hline & Mean & SD & Mean & SD & \\
\hline Age (years) & 67.0 & 9.7 & 65.6 & 7.0 & 0.36 \\
\hline Duration of menopause (years) & 19.7 & 10.4 & 16.2 & 7.7 & 0.13 \\
\hline Femoral neck BMD $\left(\mathrm{g} / \mathrm{cm}^{2}\right)$ & 0.57 & 0.09 & 0.66 & 0.12 & $<0.001$ \\
\hline Spine BMD $\left(\mathrm{g} / \mathrm{cm}^{2}\right)$ & 0.64 & 0.12 & 0.75 & 0.15 & 0.003 \\
\hline Femoral neck T-score & -3.6 & 0.76 & -2.9 & 0.83 & $<0.001$ \\
\hline Spine T-score & -2.7 & 0.79 & -2.1 & 0.95 & $<0.001$ \\
\hline Femoral neck Z-score & -1.5 & 0.80 & -0.8 & 0.67 & $<0.001$ \\
\hline Spine Z-score & -1.9 & 0.66 & -1.2 & 0.84 & $<0.001$ \\
\hline
\end{tabular}

${ }^{a}$ Student $t$-test.

$S D=$ standard deviation . 
mass ( $\mathrm{T}$-score $<-2.5$, as recommended by $\mathrm{WHO}$ ) at the femoral neck was more frequent in women with fractures than at the spine $(85.3 \%$ versus $61.7 \%$, $P=0.007)$. In contrast, based on BMD Z-score $<-1$, low bone mass at the spine was more frequent than at the femoral neck $(97.0 \%$ versus $76.4 \%, P<0.015)$.

The overall accuracy of BMD parameters (femoral neck and spine $\mathrm{BMD} \mathrm{g} / \mathrm{cm}^{2}$, T-score and Z-score) in discriminating high-risk women are presented in Table 2 in terms of the AUC and standard error (SE). All parameters showed a significant ability to recognize high-risk (fracture) patients, but femoral neck BMD Z-score yielded the highest accuracy among all the patients in discriminating fracture from nonfracture patients [AUC 0.77 (SE $0.05), P<0.001]$. This was followed by femoral neck BMD T-score [AUC 0.75 (SE 0.06), $P<0.001]$ and spine BMD Z-score [AUC 0.74 (SE 0.06), $P<0.001]$, whereas the spine BMD T-score yielded the lowest accuracy [AUC 0.71 (SE 0.62), $P=0.002$ ].

The accuracy of BMD parameters werecomparedbetween the 2 agegroups $\geq 65$ and $<65$ years with respect to AUC values. In patients aged $<65$ years, AUC values for femoral neck BMD g/ $\mathrm{cm}^{2}$ [AUC 0.87 (SE 0.07), $P=0.002$ ] and femoral neck T-score [AUC 0.82 (SE $0.08), P=0.007]$ yielded the highest and spine $\mathrm{T}$-score the lowest predictive accuracy values [AUC 0.77 (SE 0.09), $P=0.007]$. However in patients $\geq 65$ years the femoral neck T-score [AUC 0.74 (SE 0.07), $P=0.006]$ yielded the highest accuracy followed by femoral neck Z-score [AUC 0.73 (SE 0.08), P $=0.009$ ].

In patients aged $\geq 65$ years the AUC values for spine and femoral neck $\mathrm{T}$ scores decreased by $11.6 \%$ and $11.0 \%$ respectively compared with those aged $<65$ years $(P<0.01)$, whereas the decreases in spine and femoral neck BMD Z-score were $7.6 \%$ and $7.5 \%$ lower respectively $(P<0.01)$

\section{Discussion}

The present study found that in postmenopausal women with vertebral fracture, BMD values at the fracture as well as nonfracture sites were significantly lower than those without vertebral fractures. Presence of osteoporosis at both spine and femoral neck was associated with an increased risk of vertebral fracture but osteoporosis at the femoral neck was more strongly associated with vertebral fracture than at the spine. However, about $40 \%$ of vertebral fractures developed in patients with spine BMD values at the range of osteopenia.

In this study, comparison of fracture and nonfracture sites indicated higher bone density values at the fracture site using BMD T-score, whereas based on BMD Z-scores fracture sites demonstrated lower values. Furthermore, the magnitude of spine bone density difference between women with and without fracture was greater using the BMD Z-score than the BMD T-score. Moreover, spine BMD Z-score yielded a larger AUC and higher accuracy in recognizing the fracture group compared with the spine T-score. On the other hand, the BMD T-score yielded higher sensitivity for femoral neck BMD and higher diagnostic accuracy in recognizing women with fracture compared with BMD Z-score, particularly in those aged $<65$ years.

These findings indicate greater sensitivity of spine BMD Z-score compared with spine BMD $\mathrm{T}$-score in demonstrating low bone mass as well as the extent of bone loss at the spine. At present the BMD T-score is the most widely used parameter for recognizing high-risk patients as well as for planning treatment. However, the sensitivity and accuracy of this

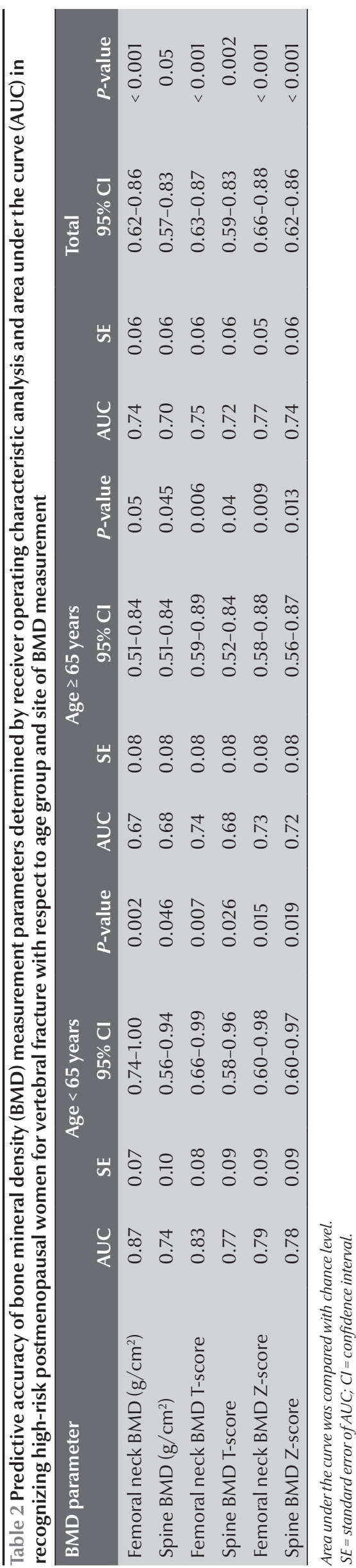


parameter varies according to age and site of measurement, whereas BMD Z-score is less affected by age and site.

Discordance in BMD T-score between the spine and femoral neck can be explained by the presence of osteoarthritis, which occurs at a higher rate in the spine than femoral neck. Additionally, accuracy and reproducibility errors which usually occur during BMD measurement may lead to discordance between sites.

Spinal osteophytosis falsely elevates BMD T-score without affecting fracture risk $[17,18]$. The impact of various forms of degenerative diseases on BMD was investigated in a study in which BMD measurement by quantitative computed tomography and DXA were compared in 209 postmenopausal women. In that study, the BMD value by posterior-anterior measurement of spine BMD via DXA (PA-DXA) was significantly higher in women with degenerative diseases changes, particularly when osteophytes were present at the vertebral bodies or facet joints, whereas BMD by lateral DXA (L-DXA) was less affected [18]. In cross-sectional studies of postmenopausal women of various age groups the accuracy of lumbar spine BMD measurement decreased with increasing age because of spinal osteoarthrosis in the elderly population [21]. The accuracy errors for spine PA-DXA can vary from $4 \%-10 \%$ and the accuracy error for proximal femur DXA measurement was estimated at $6 \%$ [22].

The amount of fat to lean body mass surrounding tissues could affect the tissue-density gradient assessed by DXA and influence the accuracy and reproducibility of BMD measurements. Postmenopausal women with greater body weight, mediated by lean body mass or fat body mass, have both higher lumbar spine, hip and femoral neck BMD and lower rate of bone loss [23]. In the present study, disproportionately higher than expected values of BMD Tscore in the spine of the fracture group compared to the femoral neck site could be attributed to the higher prevalence of osteoarthritis in the spine compared with the hip. Although we did not determine the frequency of osteoarthritis at the spine or hip, a high proportion of degenerative joint disease is expected to be present in our patients based on another study in the Islamic Republic of Iran in which spine degenerative diseases were found in $16.6 \%$ of the urban population aged 15 years and over, and hip osteoarthritis in only $0.32 \%$ of the 10921 study population [24]. A low prevalence of hip osteoarthritis was also reported in other studies [25,26], whereas arthritis of lumbar facet joints is a common radiographicfinding which has been linked to low back pain. In a study of 647 cadaveric human lumbar spines examined by a single examiner, facet arthrosis was a universal finding in $100 \%$ of patients aged 60 years and over [27].

Low sensitivity of BMD T-score was also shown in a study of 74 men from the MINOS study. In that study, $27 \%-47 \%$ ofincident fractures occurred in men with BMD T-scores -1 and -2 , whereas the percentage of incident fractures occurring with BMD T-score $<-2$ ranged from $13.7 \%-44.6 \%$. BMD $\mathrm{T}$-score had limited value in determining men at increased risk of fracture and its sensitivity for recognizing high-risk individuals was low [28].

Site-discordant fracture risk, as seen in the present study was also observed in a study of 200 men with mean age of 54.7 years. In that study, a $\mathrm{T}$-score $<-2.5$ in the femoral neck was associated with a 2.7-fold increase in the risk of vertebral fracture, while a T-score $<-2.5$ in the spine was associated with only a 2 -fold increase in risk. Both femoral neck and spine BMD were significant predictors of the presence of a vertebral fracture but the femoral area was the best site for BMD measurements [16].

The sensitivity and accuracy of BMD parameters have not been compared in any previous studies. The findings of the present study provide new data and extend the evidence-based knowledge about the utility of BMD Z-score in discriminating between the fracture and the nonfracture group. Based on our findings, the BMD Z-score may be a useful tool, not only for BMD assessment, but also for recognizing high-risk patients. In addition, our findings are consistent with the results of a previous study concerning the accuracy of femoral neck $\mathrm{T}$-score in recognizing postmenopausal women at high risk for vertebral fracture [16].

The limitations of our study should be considered. The study sample was small and the study design did not permit us to perform statistical analysis for prediction of future fractures. However, to compensate for the small sample we employed appropriate tools for characterizing the utility of BMD for discriminating fracture and nonfracture sites.

Another issue is that the data provided in this study were susceptible to information bias with respect to bone density measurement at the time of vertebral fracture as well as early performance of radiographic intervention at the time of presentation with acute back pain. Despite the clinical criteria for sample selection, we could not be certain that the vertebral fractures under review were incident fractures rather than prevalent fractures with symptom exacerbation. However, development of acute back pain and presence of localized tenderness at the site of the fracture are evidence in favour of their being incident fractures.

The focus of this study was to determine the contributive role of BMD in the development of vertebral fracture, so the influence of other factors that might be responsible for the occurrence of vertebral fractures were not considered in the statistical analysis. On the other hand, since both the fracture and the nonfracture groups were drawn from a population of the same geographic location with similar racial and cultural characteristics, the influence of other 
factors would be expected to affect both fracture and non-fracture groups equally.

In conclusion, the findings of this study indicate that postmenopausal women with vertebral fracture have significantly lower BMD values at the spine as well as at the femoral neck compared with women without fracture. Since the presence of degenerative disease in the elderly population leads to false elevation of spine BMD in postmenopausal women with vertebral fractures, BMD Z-score may be more sensitive and diagnostic than BMD T-score both for detecting low bone mass and for recognizing women at high risk of fracture. However, femoral neck BMD $\mathrm{T}$-score is less affected by degenerative joint disease and remains highly sensitive with greater accuracy in recognizing high-risk women. The results of this study require to be confirmed by further studies with larger samples.

\section{References}

1. Nevitt MC et al. The association of radiographically detected vertebral fractures with back pain and function: a prospective study. Annals of Internal Medicine, 1998, 15, 128:793-800.

2. $\mathrm{O}^{\prime} \mathrm{Nell}$ TW et al. Back pain, disability, and radiographic vertebral fractures in European women: a prospective study. Osteoporosis International, 2004, 15:760-765.

3. Cockerill $W$ et al. Health-related quality of life and radiographic vertebral fractures. Osteoporosis International, 2004, 15:113-119.

4. Fujivara $\mathrm{S}$ et al. Fracture prediction from bone mineral density in Japanese men and women. Journal of Bone and Mineral Research, 2003, 18:1547-1553.

5. Johnell $\mathrm{O}$ et al. Predictive value of BMD for hip and other fractures Journal of Bone and Mineral Research, 200, 20:1185-1194.

6. VanStaa TP et al. Bone density threshold and other predictors of vertebral fractures in patients receiving oral glucocorticotherapy. Arthritis and Rheumatism, 2003, 48:3224-3229.

7. Nevitt MC et al. Association of prevalent vertebral fractures, bone density, and alendronate treatment with incident vertebral fractures: effect of number and spinal location of fractures. The Fracture Intervention Trial Research Group. Bone, 1999, 25:613-619.

8. Nguven TV, Center JR, Fisman JA. Femoral neck bone loss predicts fracture risk independent of baseline BMD. Journal of Bone and Mineral Research, 2005, 20:1195-1201.

9. Ismail AA et al. Prevalent vertebral deformity predicts incident hip though not distal forearm fracture: results from the European Prospective Osteoporosis Study. Osteoporosis International, 2001, 12:85-90.

10. Kaptoge $\mathrm{S}$ et al. Whom to treat? The contribution of vertebral $\mathrm{X}$-rays to risk-based algorithms for fracture prediction. Results from the European prospective osteoporosis study. Osteoporosis International, 2006, 17:1369-1381.

11. Ettinger MP. Aging bone and osteoporosis: strategy for preventing fractures in the elderly. Archives of Internal Medicine, 2003, 163:227-246.

12. Whooley MA et al. Depression, falls, and risk of fracture in older women. Study of Osteoporotic Fracture Risk Group. Archives of Internal Medicine, 1999, 159:484-490.

13. Wilkins $\mathrm{CH}$, Birge SJ. Prevention of osteoporotic fractures in the elderly. American Journal of Medicine, 118:1190-1195.

14. Haung $C$ et al. Determinant of vertebral fracture prevalence among native Japanese women and women of Japanese decent living in Hawaii. Bone, 1996, 18:437-442.
15. Siris FS et al. Predictive value of low BMD for 1-year fracture outcomes is similar for postmenopausal women aged 50-64 and 65 and older: results from the National Osteoporosis Risk Assessment (NORA). Journal of Bone and Mineral Research, 2004, 19:1215-1220.

16. Legrand $\mathrm{F}$ et al. Bone mineral density and vertebral fracture in men. Osteoporosis International, 1990, 10:265-270.

17. Jones $\mathrm{G}$ et al. Prevalent vertebral deformities: relationship to bone mineral density and spinal osteophytosis in elderly men and women. Osteoporosis International, 1996, 6(3):233-239.

18. Yu W et al. Influence of degenerative joint disease on spinal bone mineral measurements in postmenopausal women. Calcified Tissue International, 1995, 57:169-174.

19. Assessment of fracture risk and its application to screening for postmenopausal osteoporosis. Report of a WHO Study Group. World Health Organization Technical Report Series, 1994, 843:1-129.

20. Genant HK, Jergas M. Assessment of prevalent and incident vertebral fractures in osteoporosis research. Osteoporosis International, 2003, 14(Suppl. 3):S43-S55.

21. Burger $\mathrm{H}$ et al. The association between age and bone mineral density in men and women aged 55 years and over: The Rotterdam Study. Bone and Mineral, 1994, 25:1-13.

22. Sarkar $\mathrm{S}$ et al. Relationship between bone mineral density and incident vertebral fracture risk and raloxifen therapy Journal of Bone and Mineral Research, 2002, 17:1-10.

23. Khosla $\mathrm{S}$ et al. Relationship between body composition and bone mass in women. Journal of Bone and Mineral Research, 1996, 11:857-863.

24. Davatchi $F$ et al. WHO-ILAR COPCORD Study (stage 1, urban study) in Iran. Journal of Rheumatology, 2008, 35:1384.

25. Andrianakos AA et al. Prevalence of symptomatic knee, hand, and hip osteoarthritis in Greece. The ESORRDIC study. Journal of Rheumatology, 2006, 32:2507-2513.

26. Chiwanichsiri D, Jimworakul A, Jitapunkul S. Lumbar disc degeneration in Thai elderly: a population-based study. Journal of the Medical Association of Thailand, 2007, 90:2477-2481.

27. Eubanks JD et al. Prevalence of lumbar facet arthrosis and its relationship to age, sex, and race: an anatomic study of cadavric specimens. Spine, 2007, 32:2058-2062.

28. Szule $\mathrm{P}$ et al. Bone mineral density predicts osteoporotic fractures in elderly men: the MINOS study. Osteoporosis International, 2005, 16:1184-1192. 